\title{
Hinged Dissections Exist
}

\author{
Timothy G. Abbott \\ MIT Computer Science and \\ Artificial Intelligence Lab \\ 32 Vassar St. \\ Cambridge, MA 02139, USA \\ tabbott@mit.edu \\ Erik D. Demaine ${ }^{\ddagger}$ \\ MIT Computer Science and \\ Artificial Intelligence Lab \\ 32 Vassar St. \\ Cambridge, MA 02139, USA \\ edemaine@mit.edu
}

\author{
Zachary Abel ${ }^{\dagger}$ \\ Dept. of Mathematics \\ Harvard University \\ 1 Oxford Street \\ Cambridge, MA 02138, USA \\ zabel@fas.harvard.edu \\ Martin L. Demaine \\ MIT Computer Science and \\ Artificial Intelligence Lab \\ 32 Vassar St. \\ Cambridge, MA 02139, USA \\ mdemaine@mit.edu
}

\author{
David Charlton \\ Dept. of Computer Science \\ Boston University \\ 111 Cummington St. \\ Boston, MA 02215, USA \\ charlton@cs.bu.edu \\ Scott D. Kominers \\ Dept. of Mathematics \\ Harvard University \\ 1 Oxford Street \\ Cambridge, MA 02138, USA \\ kominers@fas.harvard.edu
}

\begin{abstract}
We prove that any finite collection of polygons of equal area has a common hinged dissection, that is, a chain of polygons hinged at vertices that can be folded in the plane continuously without self-intersection to form any polygon in the collection. This result settles the open problem about the existence of hinged dissections between pairs of polygons that goes back implicitly to 1864 and has been studied extensively in the past ten years. Our result generalizes and indeed builds upon the result from 1814 that polygons have common dissections (without hinges). We also extend our result to edge-hinged dissections of solid 3D polyhedra that have a common (unhinged) dissection, as determined by Dehn's 1900 solution to Hilbert's Third Problem. Our proofs are constructive, giving explicit algorithms in all cases. For a constant number of planar polygons, both the number of pieces and running time required by our construction are pseudopolynomial. This bound is the best possible even for unhinged dissections. Hinged dissections have possible applications to reconfigurable robotics, programmable matter, and nanomanufacturing.
\end{abstract}

\footnotetext{
*Partially supported by an NSF Graduate Research Fellowship and an MIT-Akamai Presidential Fellowship.

${ }^{\dagger}$ Corresponding author.

†Partially supported by NSF CAREER award CCF0347776, DOE grant DE-FG02-04ER25647, and AFOSR grant FA9550-07-1-0538.
}

Permission to make digital or hard copies of all or part of this work for personal or classroom use is granted without fee provided that copies are not made or distributed for profit or commercial advantage and that copies bear this notice and the full citation on the first page. To copy otherwise, to republish, to post on servers or to redistribute to lists, requires prior specific permission and/or a fee.

SCG'08, June 9-11, 2008, College Park, Maryland, USA.

Copyright 2008 ACM 978-1-60558-071-5/08/06 ...\$5.00.

\section{Categories and Subject Descriptors}

F.2.2 [Nonnumerical Algorithms and Problems]: Geometrical problems and computations

\section{General Terms}

Algorithms, Theory

\section{INTRODUCTION}

Around 1808, Wallace asked whether every two polygons of the same area have a common dissection, that is, whether any two equalarea polygons can be cut into a finite set of congruent polygonal pieces [18, p. 222]. Figure 11 shows a simple
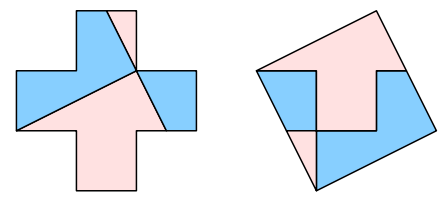

Figure 1: 4-piece dissection of Greek cross to square from 1890 [25].

\section{example. Lowry 27}

published the first solution to Wallace's problem in 1814, although Wallace may have also had a solution at the time; he published one in 1831 [36]. Shortly thereafter, Bolyai [4] and Gerwien 20] rediscovered the result, causing this result to be known sometimes as the Bolyai-Gerwien Theorem.

By contrast, Dehn 11] proved in 1900 that not all polyhedra of the same volume have a common dissection, solving Hilbert's Third Problem posed in the same year 11. Sydler 35. showed that Dehn's invariant in fact characterizes 3D dissectability.

Lowry's 2D dissection construction, as described by Frederickson 18, is particularly elegant and uses a pseudopolynomial number of pieces ${ }^{1}$ A pseudopolynomial bound is the best possible in the worst case: dissecting a polygon of

\footnotetext{
${ }^{1}$ In a geometric context, pseudopolynomial means polynomial in the combinatorial complexity $(n)$ and the dimensions of the integer grid on which the input is drawn. Although the construction does not require the vertices to have rational coordinates, a pseudopolynomial analysis makes sense only in this case.
} 


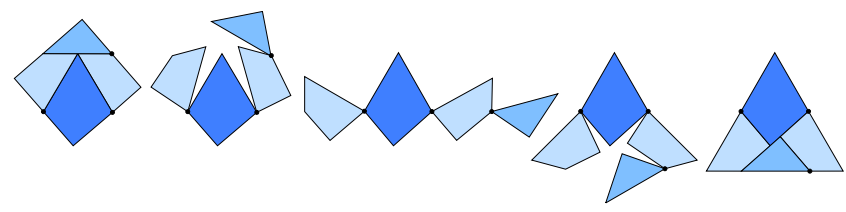

Figure 2: Dudeney's 1902 hinged dissection of a square into a triangle 15$]$.

diameter $x>1$ into a polygon of diameter 1 (for example, a long skinny triangle into an equilateral triangle) requires at least $x$ pieces. With this worst-case result in hand, attention has turned to optimal dissections using the fewest pieces possible for the two given polygons. This problem has been studied extensively for centuries in the mathematics literature $31,7,18$ and the puzzle literature 32,25 , 28,26 , and more recently in the computational geometry literature $10,24,3]$.

Hinged dissections are dissections with an additional constraint: the polygonal pieces must be hinged together at vertices into a connected assembly. The first published hinged dissection appeared in 1864, illustrating Euclid's Proposition I.47 23]; see 19 pp. 4-5]. The most famous hinged dissection is Dudeney's from 1902 [15]; see Figure 2 This surprising construction has inspired many to investigate hinged dissections; see, for example, Frederickson's book 19.

Yet the fundamental problem of general hinged dissection has remained open 14, 30: do every two polygons of the same area have a common hinged dissection? This problem has been attacked in the computational geometry literature [2, 12, 13, 17], but has been solved only in special cases. For example, all polygons made from edge-to-edge gluings of $n$ identical subpolygons (such as polyominoes) have been shown to have a common hinged dissection 12 . Perhaps most intriguingly, Eppstein [17. showed that finding a common hinged dissection of any two triangles of equal area is just as hard as the general problem.

Hinged dissections are intriguing from the perspectives of reconfigurable robotics, programmable matter, and nanomanufacturing. Recent progress has enabled chemists to build millimeter-scale "self-working" $2 \mathrm{D}$ hinged dissections such as Dudeney's 29]. An analog for 3D hinged dissections may enable the building of a complex $3 \mathrm{D}$ structure out of a chain of units; see 21 for one such approach. We could even envision an object that can re-assemble itself into different 3D structures on demand 13 . This approach contrasts existing approaches to reconfigurable robotics (see, for example, 33]), where units must reconfigure by attaching and detaching from each other through a complicated mechanism.

Our results. We settle the hinged dissection open problem, first formally posed in a CCCG 1999 paper 12 but implicit back to 1864 23] and 1902 15. Specifically, Section 3 proves a universality result: any two polygons of the same area have a common hinged dissection. In fact, our result is stronger, building a single hinged dissection that can fold into any finite set of desired polygons of the same area. The analogous multipolygon result for (unhinged) dissections is obvioussimply overlay the pairwise dissections - but no such general combination technique is known for hinged dissections. Indeed, the lack of such a transitivity construction has been the main challenge in constructing general hinged dissections.

Our construction starts from an arbitrary (unhinged) dissection, such as Lowry's 27. We show that any dissection of a finite set of polygons can be subdivided and hinged so that the resulting hinged dissection folds into all of the original polygons. We give a method of subdividing pieces of a hinged figure which effectively allows us to "unhinge" a portion of the figure and "re-attach" it at an alternate location. This construction allows us to "move" pieces and hinges around arbitrarily, at the cost of extra pieces. Thus we are able to hinge any dissection.

This initial construction easily leads to an exponential number of pieces, but we show in Section 5 that a more careful execution of Lowry's dissection 27 attains a pseudopolynomial number of pieces for a constant number of target polygons. As mentioned above, such a bound is essentially best possible, even for unhinged dissections (though we likely do not obtain the optimal constant exponent). This more efficient construction requires significantly more complex gadgets for simultaneously moving several groups of pieces at roughly the same cost as moving a single piece, and relies on specific properties of Lowry's dissection.

We also solve another open problem concerning the precise model of hinged dissections. In perhaps the most natural model of hinged dissections, pieces cannot properly overlap during the folding motion from one configuration to another. However, all theoretical work concerning hinged dissections $2,12,13,17$, has only been able to analyze the "wobbly hinged" model 19, where pieces may intersect during the motion. Is there a difference between these two models? Again this problem was first formally posed at CCCG 1999 12. We prove in Section 4 that any wobbly hinged dissection can be subdivided to enable continuous motions without piece intersection, at the cost of increasing the combinatorial complexity of the hinged dissection by only a constant factor. This result builds on expansive motions from the Carpenter's Rule Theorem 9, 34 combined with the theory of slender adornments from SoCG 2006 8.

The following theorem summarizes our results in $2 \mathrm{D}$ :

THEOREM 1. Any finite set of polygons of equal area have a common hinged dissection which can fold continuously without intersection between the polygons. For a constant number of target polygons with vertices drawn on a rational grid, the number of pieces is pseudopolynomial, as is the algorithm to compute the common hinged dissection.

Finally, we generalize our results to 3D in Section 6. As mentioned above, not all 3D polyhedra have a common dissection even without hinges. Our techniques generalize to show that hinged dissections exist whenever dissections do:

TheOREM 2. Any finite set of $3 D$ polyhedra with the same volume and Dehn invariant have a common hinged dissection.

\section{TERMINOLOGY}

A hinged figure $F$ is a finite collection of simple, oriented polygons (the links) hinged together at rotatable joints at the links' vertices so that the resulting figure is connected, together with a fixed cyclic order of links around each hinge. (Note that a hinge might exist at a $180^{\circ}$ angle of a link, 


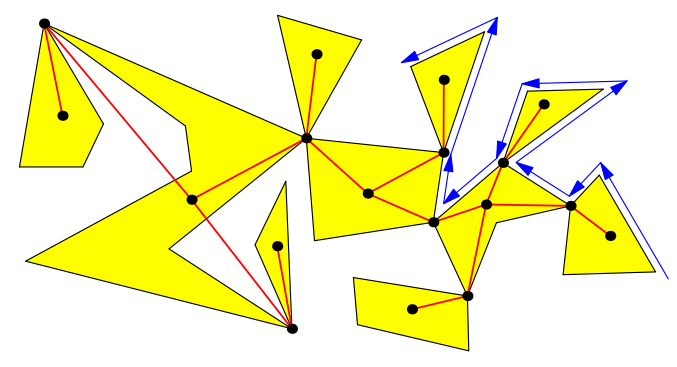

Figure 3: A hinged figure, its incidence graph, and part of its boundary path.

but this hinge is still considered a vertex of the link.) A configuration of a hinged figure $F$ is an embedding of $F$ 's links into the plane so that the links' interiors are disjoint and so that each hinge's cyclic link order is maintained.

The incidence graph of a hinged figure is the graph that has a vertex corresponding to every link and every hinge, such that two nodes are connected by an edge if one represents a link and the other represents a hinge on that link. See Figure 3 A hinged figure is tree-like if the incidence graph is a tree, and it is chain-like if the incidence graph is an open chain.

The boundary $\partial A$ of a hinged figure $A$ is the oriented path (or collection of paths) along the edges of the links traversed in depth-first order, as illustrated in Figure 3 For a tree- or chain-like figure, the boundary consists of a single path incorporating all edges of the links. Note that the boundary path will trace each hinge point multiple times, but we distinguish these as different boundary points.

For two hinged figures $A$ and $B$, we say that $B$ is a refinement of $A$, and write $B \prec A$, if $A$ can be obtained from $B$ by adding hinges between pieces of $B$, which may effectively glue together shared edges of pieces in $B$. The added hinges in the definition gives rise to an imposed configuration of $B$ for every configuration of $A$. The property of refinement is transitive; that is, if $C \prec B$ and $B \prec A$, then $C \prec A$. This transitivity of refinement plays a central role in the arguments below.

\section{UNIVERSAL HINGED DISSECTION}

In this section, we show that any finite collection of equalarea polygons has a common hinged dissection. More precisely, we construct a hinged figure with a configuration in the shape of every desired polygon; continuous motions without intersection will be addressed in Section 4. The proof is in three parts: effectively moving rooted subtrees, effectively moving rooted supersubtrees, and arbitrarily rearranging supertrees.

\subsection{Moving Rooted Subtrees}

Consider a tree-like hinged figure $F$. If there are two hinged figures $A$ and $B$ with two distinguished boundary points $a \in \partial A$ and $b \in \partial B$ so that $F$ is equivalent to the hinged figure obtained by identifying points $a$ and $b$ to a single hinge (denoted $F=A_{(a)} \wedge_{(b)} B$ ), then we say $A$ and $B$ are each rooted subtrees of $F$. If another boundary point $b^{\prime} \in \partial B$ is chosen, then the new hinged figure $F^{\prime}=A_{(a)} \wedge_{\left(b^{\prime}\right)} B$ is related to $F$ by a rooted subtree movement: $(A, a)$ is the subtree that has been moved.

Our goal is to accomplish this movement with hinged dis-

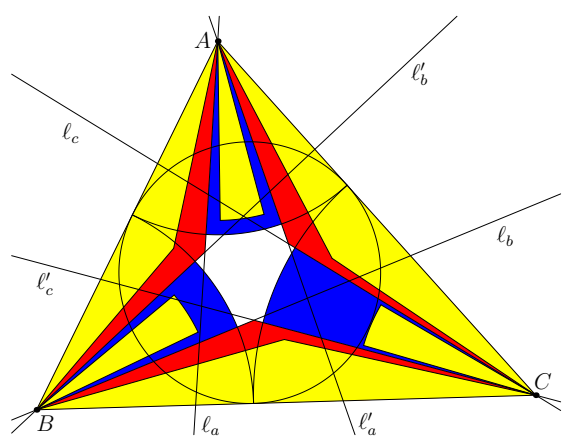

Figure 4: The free regions (lightly colored) in triangle $A B C$ are separated by the dark circles and the angle trisectors $\ell_{a}, \ell_{a}^{\prime}$, etc.

section. We will achieve this goal by connecting pieces with chains of isosceles triangles hinged at their base vertices. We begin with a lemma concerning cutting isosceles triangles from polygons, and then proceed to construct the required dissection by cutting out chains from both $A$ (at the point a) and $B$ (along the boundary from $b$ to $b^{\prime}$ ).

For an angle $\alpha<90^{\circ}$ and a length $\ell$, denote by $\mathcal{T}_{\alpha}(\ell)$ the isosceles triangle with base of length $\ell$ and base-angles $\alpha$. For a segment $P Q$, use the notation $\mathcal{T}_{\alpha}(P Q)$ for the triangle $\mathcal{T}_{\alpha}(|P Q|)$ drawn with base along segment $P Q$. Finally, for an angle $\beta$, point $P$, and radius $r$, let $\mathcal{S}_{\beta}(P, r)$ be a circular sector centered at $P$ with angle $\beta$ and radius $r$.

Lemma 3. For any simple polygon $V=V_{1} \ldots V_{n}$, there exist an angle $\beta$ and a radius $r$ small enough so that the triangles $\mathcal{T}_{\beta}\left(V_{i} V_{i+1}\right)$ constructed inward along the edges, as well as circular sectors $\mathcal{S}_{\beta}\left(V_{i}, r\right)$ drawn inside $V$, are pairwise disjoint except at the vertices of $V$. These triangles and sectors will be called the free-regions for their respective edges or vertices of $V$.

Proof. We first prove the result for triangles. For triangle $T=A B C$ with side lengths $a, b, c$, semiperimeter $s=\frac{1}{2}(a+b+c)$, and angles $\delta, \epsilon, \zeta$, choose $\beta_{T}<\frac{1}{3} \min \{\delta, \epsilon, \zeta\}$ and $r_{T}<\min \{s-a, s-b, s-c\}$. Then the triangles $\mathcal{T}_{\beta_{T}}(A B)$, etc., and the sectors $\mathcal{S}_{\beta_{T}}\left(A, r_{T}\right)$, etc., can be drawn in the triangle without overlap, as in Figure 4 Indeed, $\mathcal{T}_{\beta_{T}}(A B)$ is contained between $A B$ and the two trisectors $\ell_{a}$ and $\ell_{b}^{\prime}$ (the region shown in red), sector $\mathcal{S}_{\beta_{T}}\left(A, r_{T}\right)$ is contained in the sector $\mathcal{S}_{\frac{\delta}{3}}(A, s-a)$ between trisectors $\ell_{a}$ and $\ell_{a}^{\prime}$ (shown in green), etc., and these six regions are interior-disjoint.

For the general case, first triangulate polygon $V=$ $V_{1} \cdots V_{n}$ by $n-2$ diagonals. For each triangle $T=V_{i} V_{j} V_{k}$ in the triangulation, calculate $\beta_{T}$ and $r_{T}$ as above, and draw the free regions in $T$. Finally, as all resulting triangles and sectors are disjoint (except at vertices), choosing $\beta=\min _{T}\left\{\beta_{T}\right\}$ and $r=\min _{T}\left\{r_{T}\right\}$ suffices.

For a sequence of positive lengths $\ell_{1}, \ldots, \ell_{n}$, we define the chain $\mathcal{C}_{\alpha}\left(\ell_{1}, \ldots, \ell_{n}\right)=C$ to be the hinged figure formed by hinging the $2 n$ upward-pointing triangles

$$
\mathcal{T}_{\alpha}\left(\ell_{1}\right), \mathcal{T}_{\alpha}\left(\ell_{1}\right), \mathcal{T}_{\alpha}\left(\ell_{2}\right), \mathcal{T}_{\alpha}\left(\ell_{2}\right), \ldots, \mathcal{T}_{\alpha}\left(\ell_{n}\right), \mathcal{T}_{\alpha}\left(\ell_{n}\right)
$$

in order at their base vertices. The initial point $C_{0}$ and final point $C_{1}$ of this chain are the unhinged vertices of the first $\mathcal{T}_{\alpha}\left(s_{1}\right)$ and the last $\mathcal{T}_{\alpha}\left(s_{n}\right)$ respectively.

We now state and prove the desired result of this section. 


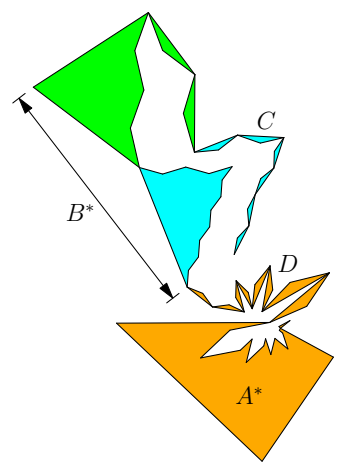

(a)

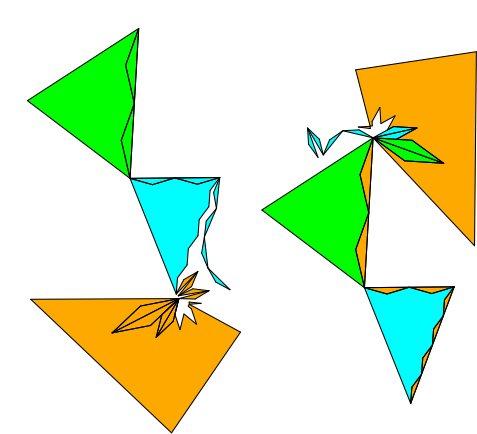

(c)

Figure 5: Effectively moving a rooted subtree

THEOREM 4. For any two tree-like figures $F$ and $F^{\prime}$ related by the rooted subtree movement of $(A, a)$ from $(B, b)$ to $\left(B, b^{\prime}\right)$, there exists a common refinement $G \prec F$ and $G \prec F^{\prime}$. Further, if a lies on link $L_{a} \in A$, and a simple path $\gamma$ along $\partial B$ is chosen from $b$ to $b^{\prime}$, this refinement $G \prec F$ may be chosen so that only $L_{a}$ and links incident with $\gamma$ are refined.

Note first that both $A$ and $B$ are tree-like, as they are subtrees of tree-like figure $F$. Note also that there are exactly two boundary paths $\gamma$ from $b$ to $b^{\prime}$ since $B$ is tree-like.

Proof. Without loss of generality, the diagram is oriented so that $\gamma$ traces the boundary of $B$ counterclockwise from $b$. The construction is in two steps.

In the first step, we cut a chain from the boundary of $\gamma$, as follows. Let $r$ be the smallest free-region radius for all links touched by $\gamma$, and likewise let $\alpha$ be the smallest freeregion angle. Path $\gamma$ is a polygonal path $P_{0} P_{1} \ldots P_{t}$ along the boundary of $B$, where $P_{i}$ are vertices of links with $P_{0}=b$ and $P_{t}=b^{\prime}$. By refining this path into shorter segments as necessary, we may assume that each segment $P_{i-1} P_{i}$ has length $2 \ell_{i}$ with $\ell_{i} \leq r$.

Choose an angle $\beta<\alpha / 2 t$. Next, cut out $2 t$ isosceles triangles along $\gamma$ : for each segment $P_{i} P_{i+1} \in \gamma$, cut two $\mathcal{T}_{\beta}\left(\ell_{i}\right)$ triangles. These triangles fit in the appropriate free-triangle for their link in $B$ by choice of $\beta$, so all of these triangles may indeed be removed without overlapping or disconnecting any of $B$ 's links. Let $B^{*}$ be the hinged figure after these triangles have been removed, and let $C=\mathcal{C}_{\beta}\left(\ell_{1}, \ldots, \ell_{t}\right)$ be the chain formed by hinging these $2 t$ cut-out triangles in order. Finally, rehinge the pieces to form the figure $G_{b}=B^{*}{ }_{\left(b^{\prime}\right)} \wedge{ }_{\left(C_{1}\right)} C$. See Figure 5 for an illustration.

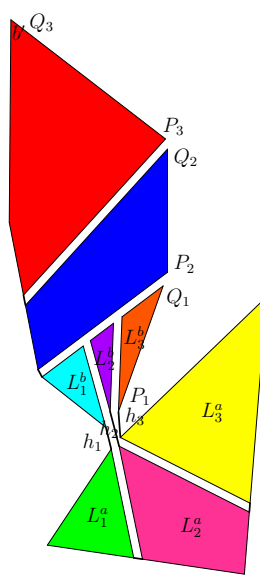

(a)

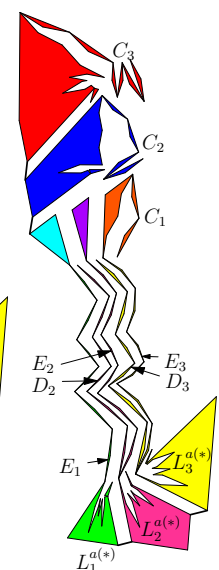

(b)

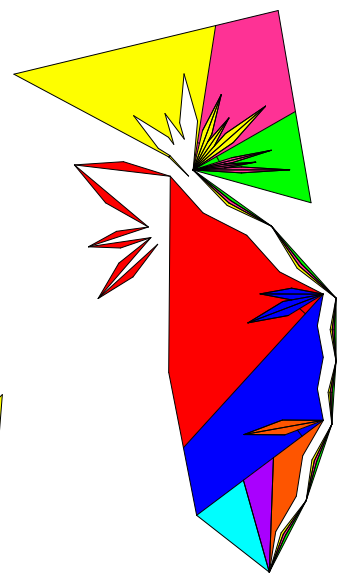

(c)
Figure 6: Moving a rooted supersubtree.

The other step is to cut a chain away from $A$ at $a$. Draw $t$ abutting rhombi $r_{1}, \ldots, r_{t}$ in link $L_{a}$ at point $a$ so that $r_{i}$ has a diagonal of length $\ell_{i}$ and an angle of $2 \beta$; they are drawn in the order $r_{1}, \ldots, r_{k}$ clockwise around $a$ so that $r_{i}$ shares (part of) an edge with $r_{i+1}$ for $1 \leq i \leq k-1$. Call this configuration of kites a kite-sweep $\mathcal{K S}_{\beta}\left(\ell_{1}, \ldots, \ell_{n}\right)$. Recall that $\beta$ was chosen so that $2 t \beta<\alpha$ and that $\ell_{i}$ were chosen so that $\ell_{i}<r$ for each $1 \leq i \leq r$, so this kite-sweep can fit within the free-sector of $L_{a}$ at $a$. Finally, cut out these $t$ rhombi in the form of $2 t \beta$-triangles, rehinging them into $D=\mathcal{C}_{\beta}\left(\ell_{1}, \ldots, \ell_{t}\right)$. Link $L_{a}$ is no longer a simple polygon, so simply cut away a small corner near $a$ and rehinge it as shown in Figure 5. Let $A^{*}$ be the remaining hinged figure after $A$ has been thus mutilated. Finally, hinge all of $A$ back together in the form $G_{a}=A^{*}{ }_{(a)} \wedge{ }_{\left(d_{1}\right)} D$.

The final result of our construction is the single hinged figure $G=G_{a\left(D_{0}\right)} \wedge{ }_{(b)} G_{b}$; we claim $G \prec F$ and $G \prec F^{\prime}$. To see $G \prec F$, simply configure chains $C$ and $D$ so that each link assumes the spot from which it was cut from $F$; i.e., chain $C$ fills the triangular holes left along path $\gamma$, and chain $D$ fills the kite-sweep in $L_{a}$. See Figure 5 (c), left. For the refinement $G \prec F^{\prime}$, the chains simply switch roles: chain $D$ now fills in the gaps left along $\gamma$, and chain $C$ fills the kite holes in $L_{a}$. See Figure 5(c), right.

\subsection{Moving Rooted Supersubtrees}

Now we increase the level of abstraction by allowing movement of rooted subtrees in a hinged figure $F$ that already has a refinement $G \prec F$. We call $F$ the superfigure of $G$, and subtrees of $F$ supersubtrees of $G$.

THEOREM 5. Take tree-like figures $F$ and $F^{\prime}$ related by the rooted-subtree movement of $(A, a)$ from $(B, b)$ to $\left(B, b^{\prime}\right)$ as in Theorem 4, and suppose $G \prec F$. Then there exists a common refinement $H \prec G \prec F$ and $H \prec F^{\prime}$. Further, if a path $\gamma$ from $b$ to $b^{\prime}$ on $\partial B$ is chosen, then only links of $G$ incident with $\gamma$ are refined.

In other words, this theorem allows the movement of a supersubtree of $G$. The construction below directly generalizes the method used in Theorem 4.

Proof. We will write from $G$ 's point of view, so features of $F$ will have the super prefix. Without loss of generality, 
suppose $\gamma$ winds counterclockwise around the superboundary of $B$.

Consider the behavior near the superhinge $h$ of $F$ corresponding to points $a$ and $b$; let $\left\{h_{i} \mid 1 \leq i \leq n\right\}$ be the set of all the hinges of $G$ with the property that $h_{i}$ has two incident links $L_{i}^{a}$ and $L_{i}^{b}$ lying in $A$ and $B$ respectively. As links are defined to be simple polygons, these $2 n$ links are distinct. We may suppose that these $2 n$ links are the only links of $G$ incident with superhinge $h$ : the construction below is unchanged by the presence of more, extraneous links. Without loss of generality, we may assume that these links have been numbered so that they fall in the cyclic order $L_{1}^{a}, \ldots, L_{n-1}^{a}, L_{n}^{a}, L_{n}^{b}, L_{n-1}^{b}, \ldots, L_{1}^{b}$ counterclockwise around $h$.

Our goal is to mimic the two steps in the proof of Theorem 4 by effectively cutting a chain from $A$ at $a$ and cutting a chain from $B$ along $\gamma$.

We begin by choosing the dimensions of the chain. First, the refinement $G \prec F$ induces an identification of some boundary points of $G$, and any point $p \in \partial G$ collocated with a vertex of any link in $G$ will itself be declared a (possibly flat) vertex of its link. We also declare $b^{\prime}$ to be a vertex of its link, if it isn't already. Let $r$ and $\alpha$ be the smallest free-region radius and angle for any link in $G$ incident with $\gamma$. Polygonal path $\gamma$ consists of $t$ segments $P_{i} Q_{i},(1 \leq i \leq t)$ from the boundary of $G$, where $P_{1}$ corresponds to $b$ and $Q_{t}$ corresponds to $b^{\prime}$; as before, we may subdivide $\gamma$ as necessary so that $\left|P_{i} Q_{i}\right|=2 \ell_{i} \leq 2 r$ for each $1 \leq i \leq n$. We choose $\beta=\alpha / 2 t$.

Note that boundary point $Q_{i}$ of $G$ does not necessarily equal $P_{i+1}$, but if they are unequal then both $Q_{i}$ and $P_{i+1}$ are vertices of their respective links; let $i_{0}, \ldots, i_{s}$ be the indices where $Q_{i_{j}-1} \neq P_{i_{j}}$, with $i_{0}=1$ and $i_{s}=t+1$.

We begin by refining the links along $\gamma$ to imitate the first step in the construction of Theorem 4 i.e. to simulate cutting a $\mathcal{C}_{\beta}\left(\ell_{1}, \ldots, \ell_{t}\right)$ from $\gamma$ and linking it onto $b^{\prime}$. We treat each portion $P_{i_{j}+1} Q_{i_{j+1}}$ of $\gamma$, corresponding to a contiguous path along $\partial G$, separately. For each $i_{j} \leq k \leq i_{j+1}-1$, cut two $\mathcal{T}_{\beta}\left(\ell_{k}\right)$ triangles inward along $P_{k} Q_{k}$. Also, cut a kite-sweep $\mathcal{K} \mathcal{S}_{\beta}\left(\ell_{1}, \ldots, \ell_{i_{j}-1}\right)$ from the free-sector at $P_{i_{j}}$, and then make the link simple by removing and rehinging a small corner as shown in Figure 6(b). Notice that, since $\beta$ is less than the free-region angle along each edge incident with $\gamma$, all of the removed isosceles triangles fit within this region. Likewise, the kite-sweep has total angle $2 \beta \cdot\left(i_{j}-1\right) \leq 2 t \beta=\alpha$ and the largest kite has diagonal $\max \left\{\ell_{1}, \ldots, \ell_{i_{j}-1}\right\} \leq r$, so the kite-sweep fits in the freesector at $P_{i_{j}}$. We have now removed $2\left(i_{j+1}-1\right)$ triangles, namely two of each length $\ell_{1}, \ldots, \ell_{i_{j+1}-1}$, which we now rehinge into a chain $C_{j}=\mathcal{C}_{\beta}\left(\ell_{1}, \ldots, \ell_{i_{j+1}-1}\right)$ and attach to $G$ by hinging $C_{j}$ 's final point to $Q_{i_{j+1}-1}$.

To see that this construction refines $G$, note that each of the $s$ chains may simply fill in the places from which they were cut. To see that this hinged figure can also serve the purpose that $B^{*}{ }_{\left(b^{\prime}\right)} \wedge{ }_{\left(c_{0}\right)} C$ serves in Theorem 4 note that each chain $C_{j}$ may fill in the kite-sweep cut at $P_{i_{j+1}}$ for $1 \leq j \leq s-1$, while $C_{j}$ is the desired chain attached at $b^{\prime}$ (Figure 6(c)).

Now we show how to refine $G$ around superhinge $h$. For each $1 \leq i \leq n$, cut a $\mathcal{K} \mathcal{S}_{i \beta / n}\left(\ell_{1}, \ldots, \ell_{k}\right)$ kite-sweep in $L_{i}^{a}$ at $h_{i}$; the resulting non-simple link has two corners at $h_{i}$, so we cut off and rehinge the more counterclockwise of the two, calling the resulting link (without this small corner)

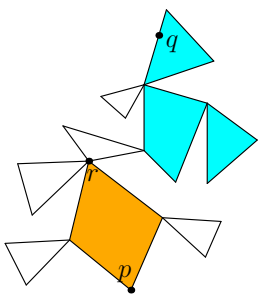

(a)

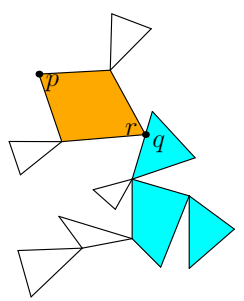

(b)

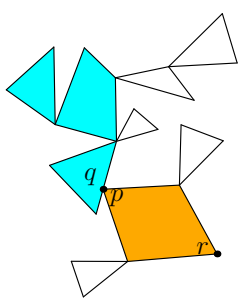

(c)
Figure 7: Rearranging a superfigure by means of rooted subtree movements.

$L_{i}^{a(*)}$. As before, by choice of $r$ and $\beta$, the $i$ th kite-sweep can fit within the free-sector of $L_{i}^{a}$ at $h_{i}$. For $2 \leq i \leq n$, cut each of the $2 t$ triangles $\mathcal{T}_{i \beta / n}\left(\ell_{j}\right)$ removed from $L_{i}^{a}$ into two pieces: a triangle $\mathcal{T}_{(i-1) \beta / n}\left(\ell_{j}\right)$ with the same base, and a kite whose four angle measures are $\beta / n, 180^{\circ}+2(i-1) \beta / n$, $\beta / n$, and $180-2 i \beta / n$. The $(i-1) \beta / n$ triangles are hinged into a chain $D_{i}^{a}=\mathcal{C}_{(i-1) \beta / n}\left(\ell_{1}, \ldots, \ell_{2 k}\right)$, and the kites are hinged into a kite-chain $E_{i}^{a}=\mathcal{C}_{(i-1) \beta / n, i \beta / n}\left(\ell_{1}, \ldots, \ell_{2 k}\right)$ as in Figure 6 for $i=1$, the $\beta / n$ triangles are hinged into the kite-chain $\left.E_{1}^{a}=\mathcal{C}_{0, i \beta / n}\left(\ell_{1}, \ldots, \ell_{2 k}\right)\right)$. We then hinge $\left(D_{i}^{a}\right)_{0}$ and $\left(E_{i}^{a}\right)_{0}$ to point $h_{i}$ of $L_{i}^{a(*)}$, and hinge point $h_{b}$ of $L_{i}^{b}$ to $E_{i}^{a}(1)$. See Figure 6 .

As before, this is a refinement of $G$ since each piece may take its original position. We now describe the alternate configuration: For $2 \leq i \leq n$, chain $D_{i}^{a}$ fills in the kitesweep of $L_{i-1}^{a}$, while $\bar{L}_{n}^{a}$ 's kite-sweep remains unfilled. The kite-chains $E_{1}, \ldots, E_{n}$ fit together to form a refinement of a chain $\mathcal{C}_{\beta}\left(\ell_{1}, \ldots, \ell_{k}\right)$ connecting the two halves. This is exactly the desired form, so we're done.

\subsection{Putting the Pieces Together}

Now we can finally write down the proof of the desired claim for this section:

THEOREM 6. For any finite collection of polygons $P_{1}, \ldots, P_{n}$ of equal area, there exists a common refinement $C \prec P_{i}$ for $1 \leq i \leq n$.

Proof Sketch. By the Lowry-Wallace-Bolyai-Gerwien Theorem, there exists a common decomposition of $P_{1}, \ldots, P_{n}$ into finitely many polygons $\left\{L_{i} \mid 1 \leq i \leq k\right\}$. Hinging these together, we may inductively apply the subtree movement constructions defined in this section as indicated in Figure 7 to obtain a full hinged dissection. For full details, see [1].

\section{CONTINUOUS MOTION}

Theorem 6 constructs a hinged dissection that has a configuration in the form of each of the $n$ polygons. This section shows how to further refine that hinged dissection to enable it to fold continuously into each polygon while avoiding intersection among the pieces:

THEOREM 7. Any hinged figure $A$ has a refinement $B \prec$ $A$ so that any two configurations of $B$ are reachable by a continuous non-self-intersecting motion.

Indeed, given polygons $P_{1}, \ldots, P_{n}$ of equal area, Theorem 6 guarantees that there exists a hinged figure $F$ that 
refines each of $P_{1}, \ldots, P_{n}$. By Theorem 7, there is a refinement $F^{\prime} \prec F$ that is universally reconfigurable without self-intersection. In particular, $F^{\prime}$ can continuously deform between any of the configurations induced by the $P_{i}$ s. This figure $F^{\prime}$ solves the problem, proving the first sentence of Theorem 1 .

To prove Theorem 7 , we require two preliminary results: the first about polygonal chains and slender adornments, and the other "chainifying" a given hinged figure.

\subsection{Slender Adornments}

Slender adornments are defined by Connelly et al. [8]. An adornment is a connected, compact region together with a line segment $a b$ (the base) lying inside the region. Furthermore, the two boundary arcs from $a$ to $b$ must be piecewise differentiable, with one-sided derivatives existing everywhere. An adornment is a slender adornment if for every point $p$ on the boundary other than $a$ and $b$, the primary inward normal(s) at $p$, namely the rays from $p$ perpendicular to the one-sided derivatives at $p$, intersect the base segment $a b$ (possibly at the endpoints). In 8, it is shown that chains of slender adornments cannot lock. Specifically, they show the following:

TheOREM 8. 8, Theorem 8] A strictly simple polygonal chain adorned with slender adornments can always be straightened or convexified.

(In a strictly simple polygonal chain, edges intersect each other only at common endpoints.) This implies that any strictly simple polygonal open chain is universally reconfigurable, because to find a continuous motion between two configurations $c_{1}$ and $c_{2}$, one may simply follow a motion from $c_{1}$ to the straightened configuration $c$, and then reverse a motion from $c_{2}$ to $c$.

\subsection{Chainification}

Next, we prove that any hinged figure has a refinement that is chain-like and simply adorned:

THEOREM 9. Any hinged figure $F$ has a chain-like refinement $G \prec F$ so that $G$ consists of a chain of equally-oriented obtuse triangles hinged at their acute-angled vertices.

Proof. First we refine $F$ to consist of a tree of triangles hinged at vertices, as follows. For each $n$-sided link $L$ with $n \geq 4$, draw a collection of triangulating diagonals. Sequentially, for each such diagonal $V_{1} V_{i}$ currently in link $V=V_{1} V_{2} \ldots V_{k}$ (which may be a refinement of an original link), replace $V$ with two links $V_{1} V_{2} \ldots V_{i}$ and $V_{i} V_{i+1} \ldots V_{1}$ hinged at $V_{1}$, attaching the hinge originally at $V_{i}$ to its corresponding position on either refined piece. The resulting figure indeed consists of triangles hinged at vertices.

Next, if the resulting triangulated figure is not tree-like, we may repeatedly remove an edge from a cycle in the incidence graph (i.e. remove the corresponding link from its hinge) until the graph becomes tree-like. Call this refinement $H$.

For each triangular link $A B C$ in $H$, divide $A B C$ into three triangles $A I B, B I C, C I A$, where $I$ is the incenter of $\triangle A B C$. Note that $\angle B I C=\pi-\frac{1}{2} \angle B-\frac{1}{2} \angle C>$ $\pi-\frac{1}{2}(\angle A+\angle B+\angle C)=\frac{\pi}{2}$, i.e. $\angle B I C$ is obtuse, and likewise for the others. Finally, by hinging these obtuse triangles at the base vertices by walking around $H$ 's boundary (Figure 8), we obtain the desired chain-like refinement $G$.

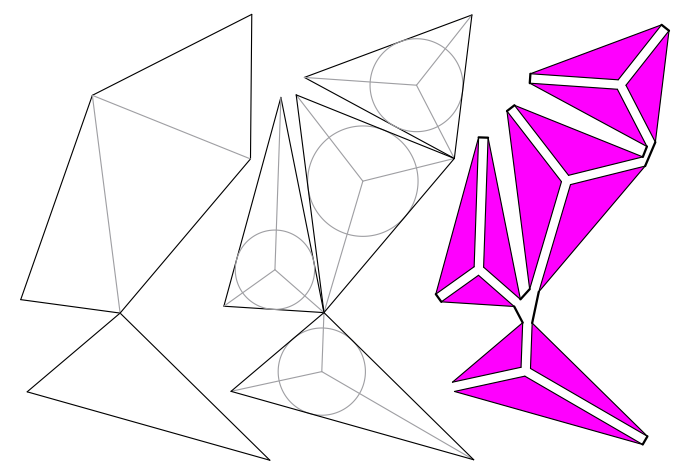

Figure 8: Chainifying a hinged figure

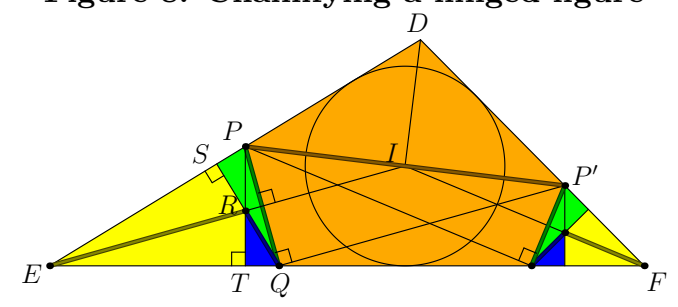

Figure 9: Refinement to hide bars from each other.

\subsection{Final Piece of the Puzzle}

We now prove Theorem 7 i.e., that any hinged figure $A$ has a universally reconfigurable refinement $B$.

Proof of Theorem 7. As shown in Theorem 9, $A$ has a refinement $C$ consisting of obtuse triangles hinged along their bases. For each such obtuse triangle $\triangle D E F$, we create the following 7-piece refinement (Figure 9p. Let $I$ be the incenter of triangle $D E F$, and suppose the line through $I$ perpendicular to $D I$ intersects sides $D E$ and $D F$ at $P$ and $P^{\prime}$ respectively; by obtuseness of $D I E, P$ lies on the interior of side $D E$, and likewise for $P^{\prime}$. Reflect $P$ over angle bisector $E I$ to $Q$; it is not hard to check that $\angle P Q P^{\prime}=90$. Define $R, S$, and $T$ as illustrated; since $P E Q$ is isosceles and acute, $R$ is inside $P E Q$. Repeat on the other side to form the 7piece refinement as illustrated. As the angles in all of the adornments are $90^{\circ}$ or larger, each can be easily checked to be slender. Furthermore, no bar can touch any other except at the vertices, since the bars in $D E F$ only touch the boundary of $D E F$ at single vertices, and no two bars within $D E F$ are touching. Thus, the resulting hinged figure $B$ is a strictly simple polygonal chain with slender adornments that refines $C$ (and hence refines $A$ ), so we are done.

\section{PSEUDOPOLYNOMIAL}

We now describe how to combine the preceding steps with ideas of Eppstein 17] and the classical rectangle-to-rectangle dissection of Montucla 31 to perform our hinged dissection using only a pseudopolynomial number of pieces, proving the second sentence of Theorem 1 In contrast to Theorem 6 we will only describe the transformation between two given polygons rather than arbitrarily many. A simple induction shows that the construction remains pseudopolynomial for a constant number of target polygons.

The idea is as follows: the inefficiency in the preceding construction is because movements may traverse the same 


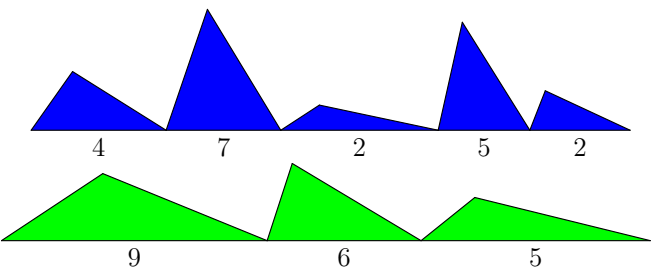

(a)

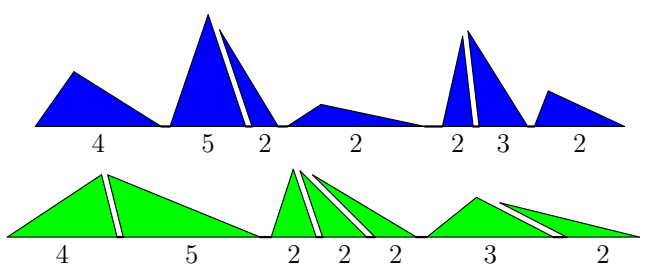

(b)

Figure 10: Equalizing the areas of two triangular chains.

hinges many times, leading to a recursive application of supersubtree movement and giving exponentially many interconnections. By performing some simplifying steps prior to subtree movement, we can instead ensure that movements are along mostly-disjoint paths so that all recursion is constant-depth.

To do this, given two figures, chainify them so we have two chains of triangles. We then further subdivide them so that both chains have the same number of links, and such that corresponding triangles have the same area. We do this using an idea from 17]: cut the triangles from base to apex along the lines that yield the desired area, hinging at the base to maintain connectivity (see Figure 10.

Given these compatible chains, our task reduces to producing hinged dissections between each pair of equal-area triangles in such a way that the base vertices of one map to the base vertices of the other. If each individual pair of triangles requires only pseudopolynomially many pieces, we will be done.

\subsection{Supercuts}

Let $G \prec F$ be hinged figures. If we make a cut in $F$, producing $F^{\prime}$, we may not be able to directly make the same cut in $G$ : attempting to do so may disconnect the figure. We give here a construction allowing us to produce an $H$ refining both $G$ and $F^{\prime}$. In keeping with earlier terminology, we call the cut in $F$ a supercut with respect to $G$. This operation will be useful in keeping everything pseudopolynomial.

TheOREM 10. Let $f_{1}$ and $f_{2}$ be boundary points along some link of a tree-like figure $F$. Let $F^{\prime}$ be the tree-like figure obtained by adding a straight-line cut between $f_{1}$ and $f_{2}$ and hinging at $f_{1}$, and suppose $G \prec F$. Then there exists a common refinement $H \prec G$ and $H \prec F^{\prime}$. Further, $H$ differs from $G$ only within the free region of the boundaries defined by adding the straight-line cut of $F^{\prime}$ to $G$.

Proof. Consider the behavior of $G$ along the edge from $f_{1}$ to $f_{2}$. In $G$ the supercut may traverse several hinged pieces. Suppose first that the supercut hits no existing hinges. Let $\left\{h_{i} \mid 1 \leq i \leq n\right\}$ be the points of intersection between the supercut and the existing edges of $G$ (so

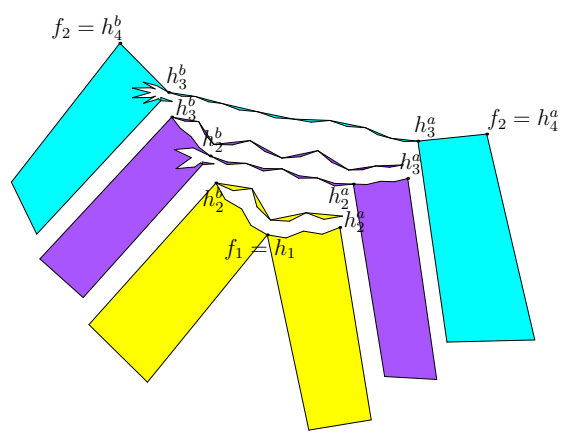

(a)

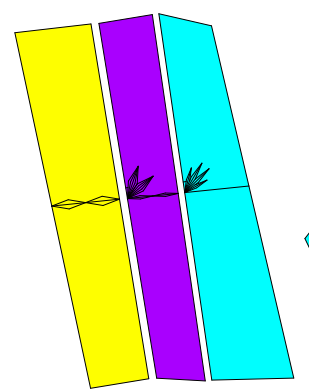

(b)

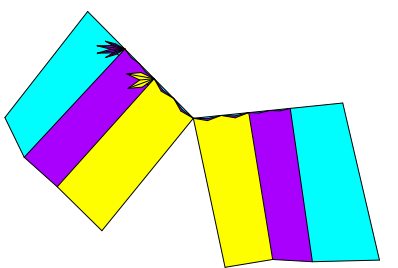

(c)
Figure 11: Making a supercut across existing edges

that in particular $h_{1}=f_{1}$ and $h_{n}=f_{2}$ ). After the cut has been made, distinguish identified vertices on each side as $h_{i}^{a}$ and $h_{i}^{b}$. We proceed inductively along the segments in $H$, beginning with $h_{1}$ to $h_{2}$ which we can easily cut and hinge exactly as in $F$.

Now suppose we have already modified all segments up to $h_{i}$ to refine $F$ appropriately. Cut the segment from $h_{i}^{a}$ to $h_{i+1}$, hinging at $h_{i}^{a}$, and perform a rooted subtree movement from $h_{i}^{a}$ to $h_{i}^{b}$. We modify this movement in two ways: first, instead of tracing the entire exterior path between the two points, we use only the direct path along the cut line, with the intermediate vertices as base points of our triangle chain. Second, since this path will cross the paths used by previous segments, we reuse all base points from earlier cutout triangles, decreasing the angle slightly to separate them; see Figure 11 for an example of this construction. Repeating this for all segments, we obtain the full supercut as desired.

Now consider the case where one or more hinges of $G$ lie on the cut edge. We only need that our inductive step can cut hinges as well as simple links. Where before our inductive transformation was based on subtree movement, for this case we will use supersubtree movement. Since we have already covered cutting links, we may here consider only links entirely on one side of the cut line. Treat all such links as a rooted supersubtree and again perform supersubtree movement traversing only the cut path instead of the entire figure boundary, and again reusing previous boundary triangle base points. The rest of the argument is identical.

Combining these two inductive steps allows us to produce the desired refinement across any existing configuration of the cut line in $G$, so we are done.

\subsection{Rectangle to Rectangle}

Our construction also requires an efficient transformation 


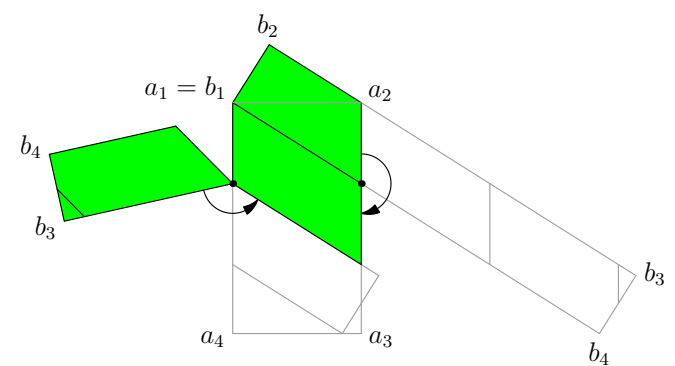

(a) Swinging $B$ back and forth to cover $A$

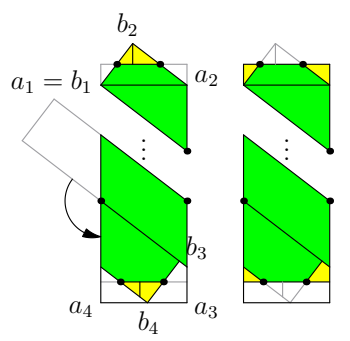

(b) Capping the top and bottom of $B$

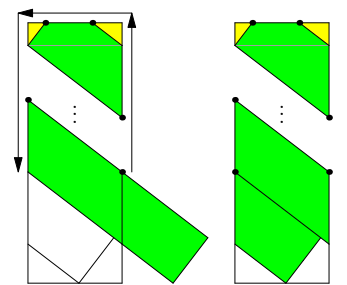

(c) Moving the end of $B$ to "wrap around" $A$

Figure 12: The stages of the rectangle-to-rectangle transformation.

between arbitrary equal-area rectangles. Since this dissection is nearly identical to the 230-year-old classical dissection, we outline this only briefly.

First (Figure 12(a)], snake the narrower rectangle $(B)$ back and forth, covering as much of the other rectangle $(A)$ as possible. Second (Figure 12(b) , cut the triangular portions of $B$ and fold them into rectangular caps, if possible. Third (Figure 12(c)), if this was not possible then we need to move the extended portion of $B$ to the left side of $A$ as if it "wrapped around" when hitting the right edge. After this, the second step is guaranteed to be successful. For full details of this construction, see 1 .

\subsection{Unaltered Subtree Movement}

It will be useful in the analysis to be able to perform subtree movements without modifying the subtree. This is in contrast to earlier constructions, which cut the kite tree out of the subtree being moved.

Accomplishing this is a simple modification of the earlier operation: we cut both the kite sweep and the triangle chain out of the free region of the parent subtree, and only need to alter the hinge connection points. The kite sweep will form a chain connecting at one end to the source vertex and at the other end to the subtree being moved, and the triangle chain will be a loose chain hanging from the source vertex (geometrically; it is not connected directly to the kite sweep); see Figure 13 To move the subtree, we then extend the kite sweep out, fill it in with the hanging triangle chain, and place the moving tree at the destination point.

\subsection{Polygon to Polygon}

With the pieces described, the transformation is simple: first, perform the equal-area chainification on both input polygons. Then convert each triangle to a rectangle using the same cutting procedure described in Figure 12(b)

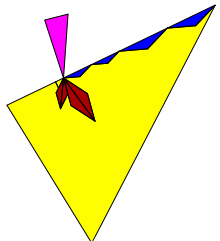

(a)

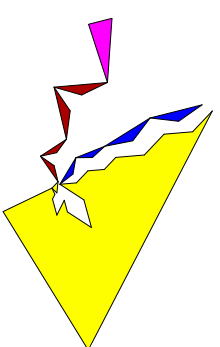

(b)

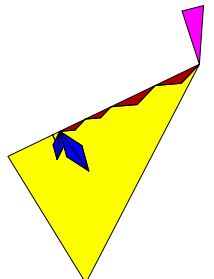

(c)
Figure 13: Moving a subtree by modifying only the parent tree.

for capping the top of the rectangle. We would then like to map between the two rectangles using the rectangle-torectangle transformation. However, to ease analysis, we actually view the rectangle-to-rectangle transformation as being done first, and then transform the rectangles back into the original triangles by making the necessary supercuts as though the figures were a solid rectangle.

After these steps, we will have pairwise dissections between the triangles in the chain. In the last step, we use Unaltered Subtree Movement to adjust the hinge joints between pairs of triangles to lie on the correct boundary points. This yields a common refinement of the two triangle chains, and we are done.

See 1] for the analysis of this procedure, as summarized by the following theorem.

THEOREM 11. The procedure described above gives a dissection with a pseudopolynomial number of pieces.

\section{THREE DIMENSIONS}

We now consider hinged figures in three dimensions. A $3 D$ hinged figure is a collection of simple polyhedra called links hinged along common positive-length edges called hinges. As before, the cyclic order of links around a hinge must remain constant.

Not every two polyhedra of equal volume have a common dissection. Dehn 11 proved an invariant that must necessarily match between the two polyhedra. For example, Dehn's invariant forbids any two distinct Platonic solids from having a common dissection. Many years later, Sydler 35 proved that polyhedra $A$ and $B$ have a common dissection if and only if $A$ and $B$ have the same volume and the same Dehn invariant. Jessen 22 simplified this proof by an algebraic technique and generalized the result to $4 \mathrm{D}$ polyhedral solids. (The 5D and higher cases remain open.) Dupont and Sah 16 gave another proof which illustrates further connections to algebraic structures.

Clearly, if two polyhedra have no common dissection, then they also have no common hinged dissection. We show the inverse: given a common dissection of polyhedra $A$ and $B$, we can construct a common hinged dissection of $A$ and $B$. More generally, we have the $3 \mathrm{D}$ analog of Theorem 6 .

Theorem 12. Given $n$ polyhedra $P_{1}, \ldots, P_{n}$ of equal volume and equal Dehn invariant, there exists a hinged figure $H$ such that $H \prec P_{i}$ for $1 \leq i \leq n$. 
Note that our algorithms assume that the (unhinged) dissection is given. None of the proofs that Dehn's invariant is sufficient are explicitly algorithmic, so it remains open whether one can compute a dissection when it exists. (We suspect, however, that this may be possible by suitable adaptation of an existing proof.)

All of the following definitions are 3D analogs of the definitions given in Section 2 The boundary $\partial A$ of a hinged figure $A$ is the 2-manifold (or collection of disjoint 2-manifolds) formed by identifying faces of links as follows: (1) for each non-hinge edge $e$ of a link $\ell$, the two faces adjacent to $e$ are connected along their common edge, and (2) for each hinge edge $e$, each pair of adjacent faces of adjacent links around $e$ are joined along their common edge. The incidence graph of a hinged figure, the notions of tree-like and chain-like, and the concept of refinement are unchanged.

The proof will be as follows: First we will describe a revised notion of free-regions for tetrahedra. Next, we illustrate the technique for moving rooted subtrees and for moving rooted supersubtrees, under the assumption that each link is a tetrahedron. By tetrahedralizing the links before each supersubtree movement, these assumptions lose nothing. The rest of the proof remains unchanged.

\subsection{Defining Free Regions}

We begin by defining free regions for a tetrahedron $T$. Choose an angle $\alpha$ smaller than the smallest dihedral angle of $T$ 's six edges. For each face $\phi$ of $T$, let free $(\phi)$ be the tetrahedron inside $T$ whose base is $\phi$ and whose base dihedral angles are $\alpha / 3$.

For each edge $e$ of $T$, construct a cylinder $C_{e}$ of length $\frac{2}{3}|e|$ centered at the midpoint of $e$ with axis along $e$. Each cylinder has radius $r$, chosen small enough so that these six cylinders do not intersect, and also so that for each edge $e$, $C_{e}$ does not intersect free ${ }_{T}\left(\phi_{3}(e)\right)$ and free ${ }_{T}\left(\phi_{4}(e)\right)$ where $\phi_{3}(e)$ and $\phi_{4}(e)$ are the faces not adjacent to $e$.

Let free $_{T}(e)$ be the wedge of $C_{e}$ of angle $\alpha / 3$ centered within the dihedral angle of $T$ at $e$. By choice of $\alpha$ and $r$, free $_{T}(e)$ will not intersect free $T(\phi)$ for any face $\phi$. These ten regions are the desired free regions for tetrahedron $T$.

\subsection{Moving Rooted Subtrees}

There are two ways to join a pair of rooted subtrees $(A, a)$ and $(B, b)$ (where $a$ and $b$ are hinges or edges of their respective figures), as each edge has two possible orientations. For each rooted subtree movement of $(A, a)$ from $(B, b)$ to $\left(B, b^{\prime}\right)$, we treat $a, b$, and $b^{\prime}$ as oriented edges, and we join them so that the orientations of the joined edges match.

We may now illustrate the analog of Theorem 4

THEOREM 13. For any two tree-like hinged figures $F$ and $F^{\prime}$ related by the rooted subtree movement of $(A, a)$ from $(B, b)$ to $\left(B, b^{\prime}\right)$ for oriented edges $a, b$, and $b^{\prime}$, there is a common refinement $G$, i.e. $G \prec F$ and $G \prec F^{\prime}$.

Proof. First we choose the boundary path. Let $p_{a}$ be the point $1 / 3$ across edge $a$, and let $\phi_{0}$ be the face of $F$ to the left of edge $a$, i.e. the face so that edge $a$ traces its boundary counterclockwise (as seen from the outside). Likewise, let $p_{b^{\prime}}$ be the point $1 / 3$ across $b^{\prime}$, and $\phi_{1}$ the face to $b^{\prime}$ 's right. Triangulate the boundary $\partial F$, and let $\gamma$ be a piecewise linear path along $\partial F$ from $p_{a}$ to $p_{b^{\prime}}$ that passes through no vertices of the triangulation, crosses each edge of each triangle orthogonally (as $\partial F$ is locally flat around

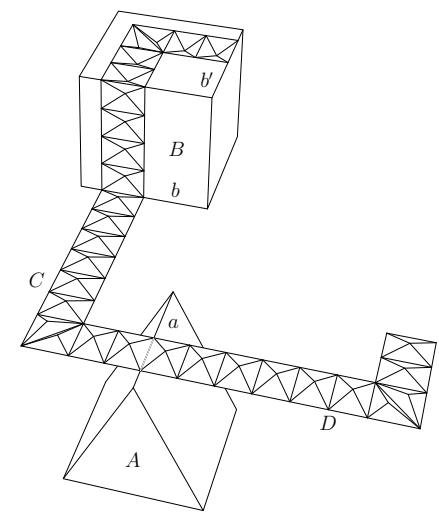

Figure 14: Moving the rooted subtree $(A, a)$ from edge $b$ to edge $b^{\prime}$.

each edge), begins in face $\phi_{0}$, and ends in face $\phi_{1}$. Without loss of generality, $\gamma$ does not cross itself, as loops may be eliminated. We may also assume that all turn angles of $\gamma$ are at most $90^{\circ}$, by truncating sharp turns.

Second, we thicken the boundary path. We now choose a small $\omega$ and form two paths $\gamma_{\ell}$ and $\gamma_{r}$ by offsetting $\gamma$ by a constant width of $\omega$ to the left and right, respectively. The value $\omega$ is chosen small enough to satisfy the following conditions: (1) $2 \omega$ is smaller than the free-radius of $a$; (2) $2 \omega<\min \{|a|,|b|\} / 3$; (3) paths $\gamma_{\ell}$ and $\gamma_{r}$ have the same number of segments as $\gamma$; (4) the region $\Gamma$ between $\gamma_{\ell}$ and $\gamma_{r}$ contains no vertices of the triangulation of $\partial F$ and does not intersect itself. In essence, we have just thickened the path $\gamma$ to have width $2 \omega$.

Third, we build a pyramid chain whose base is the region $\Gamma$, as follows. We first divide $\Gamma$ into regions that will serve as the bases. Each time $\Gamma$ crosses an edge of the triangulation of $\partial F$, draw the intersection of $\Gamma$ with this edge; it intersects $\gamma$ at right angles. At each rightward turn of $\Gamma$, let $v_{r}$ be the vertex of $\gamma_{r}$ at this turn and $v_{\ell}$ that of $\gamma_{\ell}$; draw the perpendiculars from $v_{r}$ to the two edges incident with $v_{\ell}$, as well as segment $v_{r} v_{\ell}$. Perform this procedure in mirror image for all leftward turns of $\Gamma$. These drawn segments divide $\Gamma$ into rectangles and pairs of congruent right triangles. We subdivide each such rectangle of dimensions $2 \omega \times s$ into $2 k$ rectangles of dimensions $2 \omega \times \frac{s}{2 k}$, where $k$ is chosen large enough so that $\frac{s}{2 k} \leq 2 \omega$.

We now carve out pyramids based at each of these regions along $\Gamma$. Let $\beta$ be the free region angle at edge $a$ of $A$. For some sufficiently small $h$ (to be specified soon), form for each region $R$ along $\Gamma$ the pyramid whose base is $R$ and whose vertex is at height $h$ above the center or centroid of region $R$. These pairwise-congruent pyramids hinge along their common edges to form a chain $C$ of pyramids whose base is $\Gamma$. This chain $C$ may be folded into a "kite-sweep" of octahedra and triangular bipyramids at a common segment $t$ having length $2 \omega$. If $h$ is small enough so that the total dihedral angle around $t$ is at most $\beta$, then this chain can be seen to fit within a cylindrical wedge of radius $2 \omega$, angle $\beta$, height $2 \omega$, and axis along $t$. Thus, carve out a chain $D$ duplicate to $C$ from $A$ based at the start of $\Gamma$ in this folded form. To ensure that $A$ is formed by simple polyhedra, we refine this link into a hinging of tetrahedra, possible by 6 .

Finally, hinge the mutilated $A$, the mutilated $B$, and 
chains $C$ and $D$ as illustrated in Figure 14, for the same reasons as in Theorem 4 this hinged figure forms a refinement of both $F$ and $F^{\prime}$.

\subsection{Moving Rooted Supersubtrees}

A generalization of Theorem 5 follows quickly, along the lines of our generalization of Theorem 4 above:

THEOREM 14. Take tree-like figures $F$ and $F^{\prime}$ related by the rooted-subtree movement of $(A, a)$ from $(B, b)$ to $\left(B, b^{\prime}\right)$ as in Theorem 13, and suppose $G \prec F$. Then there exists a common refinement $H$ of $G$ and $F^{\prime}$.

The rest of the proof of Theorem 12 follows the proof of Theorem 6 unchanged. We obtain Theorem 2 as an immediate corollary.

\subsection{Higher Dimensions}

We believe, though have not verified, that our techniques generalize further to refining dissections of polyhedral solids in arbitrary dimensions into equivalent hinged dissections. Again we obtain only configurations, not folding motions, for each desired polyhedral solid. Also, it is unknown when common (unhinged) dissections exist in 5D and higher 16, although the solution in $4 \mathrm{D}$ is again the Dehn invariant 22 .

Acknowledgments.. This work arose during a series of open-problem sessions for an MIT class on Geometric Folding Algorithms (6.885 in Fall 2007). We thank the other participants of those sessions for providing a productive and inspiring environment.

\section{REFERENCES}

[1] T. G. Abbott, Z. Abel, D. Charlton, E. D. Demaine, M. L. Demaine, and S. D. Kominers. Hinged dissections exist. arXiv:cs.CG/0712.2094, 2007.

http://arxiv.org/abs/0712.2094

[2] J. Akiyama and G. Nakamura. Dudeney dissection of polygons. In Revised Papers from the Japan Conf. Discrete Comput. Geom., LNCS 1763, pp. 14-29, 1998.

[3] J. Akiyama, G. Nakamura, A. Nozaki, K. Ozawa, and T. Sakai. The optimality of a certain purely recursive dissection for a sequentially $n$-divisible square. Comput. Geom. Theory Appl., 24(1):27-39, 2003.

[4] F. Bolyai. Tentamen juventutem studiosam in elementa matheseos purae, elementaris ac sublimioris, methodo intuitiva, evidentiaque huic propria, introducendi. Typis Collegii Refomatorum per Josephum et Simeonem Kali, Maros Vásárhely, 1832-1833.

[5] C. Burnikel, S. Funke, K. Mehlhorn, S. Schirra, and S. Schmitt. A separation bound for real algebraic expressions. In Proc. 9th European Sympos. Algorithms, LNCS 2161, pp. 254-265, 2001.

[6] B. Chazelle. Convex partitions of polyhedra: a lower bound and worst-case optimal algorithm. SIAM J. Comput., 13(3):488-507, 1984.

[7] M. J. Cohn. Economical triangle-square dissection. Geom. Dedicata, 3:447-467, 1975.

[8] R. Connelly, E. D. Demaine, M. L. Demaine, S. Fekete, S. Langerman, J. S. B. Mitchell, A. Ribó, and G. Rote. Locked and unlocked chains of planar shapes. In Proc. 22nd ACM Sympos. Comput. Geom., pp. 61-70, 2006.

[9] R. Connelly, E. D. Demaine, and G. Rote. Straightening polygonal arcs and convexifying polygonal cycles. Discrete Comput. Geom., 30(2):205-239, 2003.

[10] J. Czyzowicz, E. Kranakis, and J. Urrutia. Dissections, cuts, and triangulations. In Proc. 11th Canad. Conf. Comput. Geom., 1999.
[11] M. Dehn. Über den Rauminhalt. Nachrichten von der Gesellschaft der Wissenschaften zu Göttingen, Mathematisch-Physikalische Klasse, pp. 345-354, 1900. Later in Math. Ann. 55:465-478, 1902.

[12] E. D. Demaine, M. L. Demaine, D. Eppstein, G. N. Frederickson, and E. Friedman. Hinged dissection of polyominoes and polyforms. Comput. Geom. Theory Appl., 31(3):237-262, 2005.

[13] E. D. Demaine, M. L. Demaine, J. F. Lindy, and D. L. Souvaine. Hinged dissection of polypolyhedra. In Proc. 9th Workshop Algorithms Data Struct., LNCS 3608, pp. 205-217, 2005.

[14] E. D. Demaine, J. S. Mitchell, and J. O'Rourke. Problem 47: Hinged dissections. In The Open Problems Project. March 2003. http://www.cs.smith.edu/ orourke/TOPP/P47.html

[15] H. E. Dudeney. Puzzles and prizes. Weekly Dispatch, April 6-May 4, 1902.

[16] J. L. Dupont and C.-H. Sah. Homology of Euclidean groups of motions made discrete and Euclidean scissors congruences. Acta Math., 164(1):1-27, 1990.

[17] D. Eppstein. Hinged kite mirror dissection. arXiv:cs.CG/0106032, June 2001

[18] G. N. Frederickson. Dissections: Plane and Fancy. Cambridge University Press, November 1997.

[19] G. N. Frederickson. Hinged Dissections: Swinging \& Twisting. Cambridge University Press, August 2002.

[20] P. Gerwien. Zerschneidung jeder beliebigen Anzahl von gleichen geradlinigen Figuren in dieselben Stücke. J. Reine Angew. Math., 10:228-234 and Taf. III, 1833.

[21] S. Griffith. Growing Machines. PhD thesis, Media Lab, MIT, 2004.

[22] B. Jessen. The algebra of polyhedra and the Dehn-Sydler theorem. Math. Scand., 22:241-256, 1968.

[23] P. Kelland. On superposition. part II. Trans. Royal Soc. Edinburgh, 33:471-473 and plate XX, 1864.

[24] E. Kranakis, D. Krizanc, and J. Urrutia. Efficient regular polygon dissections. Geom. Dedicata, 80:247-262, 2000.

[25] D. Lemon. The Illustrated Book of Puzzles. Saxon, London, 1890.

[26] H. Lindgren. Recreational Problems in Geometric Dissections and How to Solve Them. Dover, 1972.

[27] M. Lowry. Solution to question 269, [proposed] by Mr. W. Wallace. In T. Leybourn, ed., Mathematical Repository, vol. 3, part 1, pp. 44-46. W. Glendinning, London, 1814.

[28] J. S. Madachy. Geometric dissections. In Madachy's Mathematical Recreations, ch. 1, pp. 15-33. Dover Publications, 1979.

[29] C. Mao, V. R. Thallidi, D. B. Wolfe, S. Whitesides, and G. M. Whitesides. Dissections: Self-assembled aggregates that spontaneously reconfigure their structures when their environment changes. J. Amer. Chem. Soc., 124:14508-14509, 2002.

[30] J. O'Rourke. Computational geometry column 44. Internat. J. Comput. Geom. Appl., 13(3):273-275, 2002.

[31] J. Ozanam. Récréations Mathématiques et Physiques, pp. 297-302. Claude Antoine Jombert, fils, Paris, 1778. According to 18, this material was added by J. Montucla under the pseudonym $M$. de Chanla.

[32] A.-J. Panckoucke. Les Amusemens Mathématiques. Chez André-Joseph Panckoucke, Lille, 1749.

[33] D. Rus, Z. Butler, K. Kotay, and M. Vona. Self-reconfiguring robots. Comm. ACM, 45(3):39-45, 2002.

[34] I. Streinu. Pseudo-triangulations, rigidity and motion planning. Discrete Comput. Geom., 34(4):587-635, November 2005.

[35] J.-P. Sydler. Conditions nécessaires et suffisantes pour l'équivalence des polyèdres de l'espace euclidien à trois dimensions. Comment. Math. Helv., 40:43-80, 1965.

[36] W. Wallace, editor. Elements of Geometry. Bell \& Bradfute, Edinburgh, 8th edition, 1831. 